\title{
Identification of long non-coding RNA-related and -coexpressed mRNA biomarkers for hepatocellular carcinoma
}

\author{
Fan Zhang ${ }^{1,2^{*}}$, Linda Ding ${ }^{3}$, Li Cui ${ }^{4}$, Robert Barber ${ }^{5}$ and Bin Deng ${ }^{1,2}$ \\ From The International Conference on Intelligent Biology and Medicine (ICIBM) 2018 \\ Los Angeles, CA, USA. 10-12 June 2018
}

\begin{abstract}
Background: While changes in mRNA expression during tumorigenesis have been used widely as molecular biomarkers for the diagnosis of a number of cancers, the approach has limitations. For example, traditional methods do not consider the regulatory and positional relationship between mRNA and IncRNA. The latter has been largely shown to possess tumor suppressive or oncogenic properties. The combined analysis of mRNA and IncRNA is likely to facilitate the identification of biomarkers with higher confidence.

Results: Therefore, we have developed an IncRNA-related method to identify traditional mRNA biomarkers. First we identified mRNAs that are differentially expressed in Hepatocellular Carcinoma (HCC) by comparing cancer and matched adjacent non-tumorous liver tissues. Then, we performed mRNA-IncRNA relationship and coexpression analysis and obtained 41 IncRNA-related and -coexpressed mRNA biomarkers. Next, we performed network analysis, gene ontology analysis and pathway analysis to unravel the functional roles and molecular mechanisms of these IncRNA-related and-coexpressed mRNA biomarkers. Finally, we validated the prediction and performance of the 41 IncRNA-related and -coexpressed mRNA biomarkers using Support Vector Machine model with five-fold cross-validation in an independent HCC dataset from RNA-seq.
\end{abstract}

Conclusions: Our results suggested that mRNAs expression profiles coexpressed with positionally related IncRNAs can provide important insights into early diagnosis and specific targeted gene therapy of HCC.

Keywords: Long non-coding RNA, Biomarker discovery, Hepatocellular carcinoma

\section{Background}

Hepatocellular carcinoma (HCC) is a highly prevalent, treatment-resistant malignancy with a multifaceted molecular pathogenesis and is also one of the most common cancers and the third most common cause of death by cancer worldwide [1]. Geographic areas with the highest prevalence are located in Africa and Eastern Asia, likely due to the increasing prevalence of chronic hepatitis $\mathrm{B}$ or $\mathrm{C}$. The incidence of HCC is also rising in the US. According to the American Cancer Society, an

\footnotetext{
* Correspondence: fan.zhang@uvm.edu

${ }^{1}$ Vermont Genetics Network, University of Vermont, Burlington, VT 05405 , USA

2Department of Biology, University of Vermont, Burlington, VT 05405, USA Full list of author information is available at the end of the article
}

estimated 39,230 people were diagnosed with $\mathrm{HCC}$ in the United States during 2016 and about 27,170 people will die of the cancer [2].

Although significant effort has been directed toward the improvement of surgical and medical treatment, the prognosis for patients with advanced stages of HCC remains poor [3]. To improve diagnosis and treatment efficiency, a further understanding of molecular mechanisms of HCC progression is urgently needed.

Several research strategies, high-throughput genomic microarray in particular, have been used to investigate the molecular characteristics of HCC. Many molecular biomarkers with aberrant expression have been identified in HCC tissues, including NDRG1, Importin- $\alpha 1$, FOXP1, and PTPN12. Although these findings have 
greatly improved diagnostic and therapeutic strategies, some limitations remain. First, because of the large number of mRNA and proteins in the profiles, specific targets are difficult to identify. Second, changes at the mRNA level are not always consistent with those at the protein level, suggesting that a high level of background noise may exist. Third, traditional microarray methods identify mRNAs that are differentially expressed between normal vs cancer, but don't consider the relationship between coding biomarkers and non-coding RNA, which are likely to be etiologically important.

Long noncoding RNAs (lncRNAs) are a class of noncoding RNA transcripts longer than 200 nucleotides that were previously believed to represent transcriptional noise. However, they have recently been identified as functional molecules. Emerging studies show that lncRNA play important roles in basic biology, ranging from transcriptional and post-transcriptional regulation to the control of cell cycle distribution, imprinting control, cell differentiation and tumorigenesis. Several lncRNAs have been reported to regulate the development of HCC and targets include HOXA transcription at the distal tip (HOTTIP) [4, 5], MEG3 maternally expressed 3 (MEG3) [6-8], highly upregulated in liver cancer (HULC) $[9,10]$, and ZNFX1 Antisense RNA 1 (ZFAS1) [11-13].

We hypothesized that combining mRNA expression profiles from traditional microarray methods and lncRNA expression profiles from lncRNA arrays may help identify a subset of candidate lncRNA-related and -coexpressed mRNA biomarkers with higher confidence and reliability. We defined lncRNA-related and -coexpressed mRNA biomarkers as mRNA biomarkers that are coexpressed with and have positional relationships with adjacent lncRNAs. We selected mRNA biomarkers based on two criteria: 1) differential expression between normal and cancer tissue samples; 2) a positional relationship to lncRNAs that are differentially expressed between normal and cancer samples.

In this paper, we first identify 3543 mRNA biomarkers differentially expressed between normal and cancer samples using $t$ statistics and qvalues. Then we overlapped these transcripts with the results from IncRNA arrays to identify 41 lncRNA-related and -coexpressed mRNA biomarkers. Next, we performed network analysis, functional analysis and pathway analysis for the 41 lncRNA-associated mRNA biomarkers. Finally, we used an independent dataset and a SVM model to validate the prediction performance of the 41 identified lncRNA-related and -coexpressed mRNA biomarkers. Our results showed that combined microarray and lncRNA analysis improved biomarker discovery for the treatment of HCC.

\section{Methods}

\section{IncRNA and mRNA microarray expression profiling}

We downloaded Arraystar human lncRNA and mRNA microarray data for a cohort of 16 Human HCC samples and 16 adjacent non-tumor samples from GEO (GSE58043, GSE89186, GSE64631, and GSE55191, http://www.ncbi.nlm.nih.gov/geo) [14-16]. The Arraystar human IncRNA and mRNA microarray data included five positional relationship between lncRNA and mRNA: 1) "exon sense-overlapping": the lncRNA's exon is overlapping a coding transcript exon on the same genomic strand; 2) "intron sense-overlapping": the lncRNA is overlapping the intron of a coding transcript on the same genomic strand; 3) "intronic antisense": the lncRNA is overlapping the intron of a coding transcript on the antisense strand; 4) "natural antisense": the lncRNA is transcribed from the antisense strand and overlapping with a coding transcript; and 5) "bidirectional": the lncRNA is oriented head to head to a coding transcript within $1000 \mathrm{bp}$. If the Arraystar human lncRNA and mRNA microarray data showed no overlapping or bidirectional coding transcripts nearby the lncRNA, we defined the relationship between mRNA and lncRNA as "intergenic".

\section{Statistical analysis}

We first performed a Box-Cox Power Transformation [17] using a powerTransform function (car package in $\mathrm{R}$ 3.4.0) to make the distribution of each mRNA and lncRNA in each sample approximately normal.

We used a two-sample, two-sided t-test $[18,19]$ to determine whether there was no difference between the mean of gene expression in HCC samples and that in normal samples. The null hypotheses was

$$
H_{0}: \mu_{T}=\mu_{N}
$$

where $\mu_{T}$ is the mean of gene expression in HCC samples, and $\mu_{N}$ is the mean of gene expression in normal samples. This null hypothesis was tested against the following alternative hypothesis:

$$
H_{1}: \mu_{T} \neq \mu_{N}
$$

$P$ values were determined by Welch's t-test. Qvalues were adjusted for false discovery rate control using qvalue package from Bioconductor.

\section{Pathway analysis}

Network analyses were generated through the use of Ingenuity Pathway Analysis (IPA, Redwood City, CA, USA). The top scoring network of interactions was presented for the concurrent under-expressed and the concurrent over-expressed genes. This software analyzes molecular signatures in the context of known biological 
Table 1 Forty one mRNA biomarkers with relationship to coexpressed IncRNAs (qvalue $<0.05$ for mRNA and qvalue $<0.05$ for IncRNA)

\begin{tabular}{|c|c|c|c|c|c|c|c|}
\hline mRNA & Gene & Gene expression & mRNA qvalue & IncRNA Probe name & relationship & IncRNA expression & IncRNA qvalue \\
\hline NM_000787 & $\mathrm{DBH}$ & down & 0.0153 & ASHG19A3A038177 & natural antisense & down & 0.0317 \\
\hline NM_015987 & HEBP1 & down & 0.0153 & ASHG19A3A048399 & bidirectional & down & 0.0252 \\
\hline NM_001172440 & ENDOU & down & 0.0161 & ASHG19A3A055103 & intronic antisense & down & 0.0285 \\
\hline NM_001130997 & FAM58A & up & 0.0170 & ASHG19A3A041726 & bidirectional & up & 0.0331 \\
\hline NM_000075 & CDK4 & up & 0.0216 & ASHG19A3A048765 & natural antisense & up & 0.0396 \\
\hline NM_000744 & CHRNA4 & down & 0.0218 & ASHG19A3A018571 & intronic antisense & down & 0.0285 \\
\hline NM_003074 & SMARCC1 & up & 0.0231 & ASHG19A3A022584 & intronic antisense & up & 0.0285 \\
\hline NM_025139 & ARMC9 & up & 0.0240 & ASHG19A3L0001156 & sense overlap & up & 0.0472 \\
\hline NM_014053 & FLVCR1 & up & 0.0270 & ASHG19A3A007495 & bidirectional & up & 0.0479 \\
\hline NM_000348 & SRD5A2 & down & 0.0271 & ASHG19A3L0001181 & exon sense-overlapping & down & 0.0357 \\
\hline NM_001012321 & RPSA & up & 0.0279 & ASHG19A3A020907 & natural antisense & down & 0.0293 \\
\hline NM_001334 & CTSO & down & 0.0284 & ASHG19A3A026206 & natural antisense & down & 0.0415 \\
\hline NM_030789 & HM13 & up & 0.0284 & ASHG19A3A017537 & intronic antisense & down & 0.0479 \\
\hline NM_000454 & SOD1 & down & 0.0311 & ASHG19A3A018779 & bidirectional & down & 0.0468 \\
\hline NM_002394 & SLC3A2 & up & 0.0314 & ASHG19A3A000043 & bidirectional & up & 0.0291 \\
\hline NM_144778 & MBNL2 & down & 0.0315 & ASHG19A3L0000699 & exon sense-overlapping & down & 0.0439 \\
\hline NM_001146279 & SHBG & down & 0.0315 & ASHG19A3A007528 & exon sense-overlapping & down & 0.0380 \\
\hline NM_003631 & PARG & up & 0.0328 & ASHG19A3A043936 & intronic antisense & up & 0.0289 \\
\hline NM_000182 & HADHA & down & 0.0330 & ASHG19A3A015417 & natural antisense & up & 0.0489 \\
\hline NM_003668 & MAPKAPK5 & up & 0.0333 & ASHG19A3A055106 & natural antisense & up & 0.0364 \\
\hline NM_016065 & MRPS16 & up & 0.0338 & ASHG19A3A044109 & bidirectional & up & 0.0362 \\
\hline NM_053031 & MYLK & down & 0.0338 & ASHG19A3A023105 & intronic antisense & up & 0.0285 \\
\hline NM_001040058 & SPP1 & up & 0.0355 & ASHG19A3A024471 & natural antisense & up & 0.0356 \\
\hline NM_145697 & NUF2 & up & 0.0355 & ASHG19A3A054586 & bidirectional & up & 0.0412 \\
\hline NM_172250 & MMAA & down & 0.0372 & ASHG19A3A024820 & natural antisense & down & 0.0409 \\
\hline NM_001003789 & RABL2B & down & 0.0378 & ASHG19A3A020631 & bidirectional & up & 0.0252 \\
\hline NM_001040060 & SPP1 & up & 0.0378 & ASHG19A3A024471 & natural antisense & up & 0.0356 \\
\hline NM_207304 & MBNL2 & down & 0.0379 & ASHG19A3L0000699 & exon sense-overlapping & down & 0.0439 \\
\hline NM_020791 & TAOK1 & up & 0.0382 & ASHG19A3A009329 & exon sense-overlapping & up & 0.0412 \\
\hline NM_016632 & ARL17A & up & 0.0395 & ASHG19A3A008470 & intron sense-overlapping & up & 0.0252 \\
\hline NM_014583 & LMCD1 & up & 0.0397 & ASHG19A3A020672 & bidirectional & down & 0.0311 \\
\hline NM_003937 & KYNU & down & 0.0405 & ASHG19A3A014435 & natural antisense & down & 0.0252 \\
\hline NM_000582 & SPP1 & up & 0.0418 & ASHG19A3A024471 & natural antisense & up & 0.0356 \\
\hline NM_014389 & PELP1 & up & 0.0419 & ASHG19A3A008987 & natural antisense & up & 0.0437 \\
\hline NM_148921 & EPN2 & down & 0.0433 & ASHG19A3A008042 & natural antisense & up & 0.0329 \\
\hline NM_001165031 & DTYMK & up & 0.0442 & ASHG19A3A007748 & exon sense-overlapping & up & 0.0285 \\
\hline NM_002482 & NASP & up & 0.0448 & ASHG19A3A044925 & natural antisense & up & 0.0489 \\
\hline NM_000128 & F11 & down & 0.0449 & ASHG19A3A025095 & natural antisense & down & 0.0252 \\
\hline NM_002022 & $\mathrm{FMO} 4$ & down & 0.0468 & ASHG19A3A034907 & intron sense-overlapping & down & 0.0446 \\
\hline NM_001127603 & NMRK1 & down & 0.0470 & ASHG19A3A037588 & exon sense-overlapping & down & 0.0331 \\
\hline NM_003889 & NR112 & down & 0.0471 & ASHG19A3A021464 & natural antisense & down & 0.0291 \\
\hline
\end{tabular}


Table 2 Statistics for relationships

\begin{tabular}{ll}
\hline Relationship & Counts \\
\hline Bidirectional & 9 \\
Exon sense-overlapping & 7 \\
Intron sense-overlapping & 2 \\
Intronic antisense & 6 \\
Natural antisense & 16 \\
Sense overlap & 1 \\
\hline
\end{tabular}

response and regulatory networks as well as canonical pathways.

DAVID functional analyses were used to identify biological functions that were most significantly enriched with expression changes [20].

Interpretation of biological pathways was conducted with the database we developed: Integrated Pathway Analysis Database (IPAD) (http://fzhang.w3.uvm.edu/ipad/) [21]. The enrichment scores used to select significant pathways were defined by $p$-value.

\section{Performance measurement}

We used the following five measurements for our evaluation: (1) Sensitivity (2) Specificity, (3) Precision, (4) Accuracy, and (5) Area Under the Curve.

$$
\begin{gathered}
\text { Sensitivity }=\frac{\text { true positive }}{\text { true positive }+ \text { false negative }} \\
\text { Specificity }=\frac{\text { true negative }}{\text { true positive }+ \text { false positive }} \\
\text { Precision }=\frac{\text { true positive }}{\text { true positive }+ \text { false positive }} \\
\text { Accuracy }=\frac{\text { true positive }+ \text { true negative }}{\text { true positive }+ \text { true negative }+ \text { false positive }+ \text { false negative }}
\end{gathered}
$$

\section{Results}

The Arraystar Human lncRNA and mRNA microarray profiles contained two states (tumor vs. non-tumor) with 16 samples corresponding to each state. We obtained 3543 significantly differentially expressed mRNA biomarkers (mapped to 1932 genes) with qvalue $<0.05$, among which 2066 (946 genes) were over-expressed and 1477 (986 genes) were under-expressed in HCC tumor.
Forty one mRNA biomarkers (Table 1) met our criteria of (1) being differentially expressed between 16 normal and 16 cancer samples with qualue $<0.05$; and 2) being positionally related to lncRNA which were differentially expressed between 16 normal and 16 cancer samples with qvalue $<0.05$. The information corresponding to the positional relationships of $\mathrm{mRNA} / \mathrm{miRNA}$ and lncRNAs were identified to predict the role of lncRNAs in regulating nearby genes. The positional relationship included exon sense-overlapping (7), natural antisense (16), bidirectional (9), intronic antisense(6), intron sense-overlapping (2), and sense overlap (1) (Table 2). Network analyses were performed with Ingenuity Pathway Analysis and the top four networks were identified (Table 3 and Fig. 1). We identified four networks: 1) Endocrine System Development and Function, Molecular Transport, Small Molecule Biochemistry; 2) Immunological Disease, Inflammatory Disease, Inflammatory Response; 3) Amino Acid Metabolism, Molecular Transport, Small Molecule Biochemistry; and 4) Metabolic Disease, Developmental Disorder, Hereditary Disorder. Gene ontology analysis with DAVID described the biological processes of the 41 mRNA biomarkers (Fig. 2a and b). Pathway analysis were generated using the IPAD [21] (Table 4). Pathways linked with the 41 mRNA biomarkers included Metabolism, Hemostasis, Cell Cycle, Signaling, Disease, Immune system, and Gene Expression, which are consistent with previous results we found [22-25].

In order to validate the 41 mRNA biomarkers, we built a five-fold cross-validation Support Vector Machine (SVM) model based on all the 32 samples using a radius basis function kernels function. We achieved high prediction performance $(\mathrm{AUC}=0.996$, precision $=100 \%$, accuracy $=96.9 \%, \quad$ sensitivity $=93.8 \%, \quad$ specificity $=100 \%)$. Further we randomly divided the 16 HCC samples and 16 Normal samples into two groups: training set and testing set. Each group contains $8 \mathrm{HCC}$ samples and 8 Normal samples. We used the testing test to assess the performance of the trained 41 mRNA biomarkers. The testing set was blind and no data from the testing set were used for identification of the 41 mRNA biomarkers and development of the SVM model. We obtained high performances: for the training set

\begin{tabular}{|c|c|c|}
\hline Top Diseases and Functions & Score & Focus Molecules \\
\hline Endocrine System Development and Function, Molecular Transport, Small Molecule Biochemistry & 32 & 14 \\
\hline Immunological Disease, Inflammatory Disease, Inflammatory Response & 32 & 14 \\
\hline Amino Acid Metabolism, Molecular Transport, Small Molecule Biochemistry & 21 & 10 \\
\hline Metabolic Disease, Developmental Disorder, Hereditary Disorder & 3 & 1 \\
\hline
\end{tabular}
$(\mathrm{AUC}=1.0$, precision $=100 \%$, accuracy $=93.8 \%$, sensitivity $=87.5 \%$, specificity $=100 \%$ ) and for testing set

Table 3 Top networks involved 


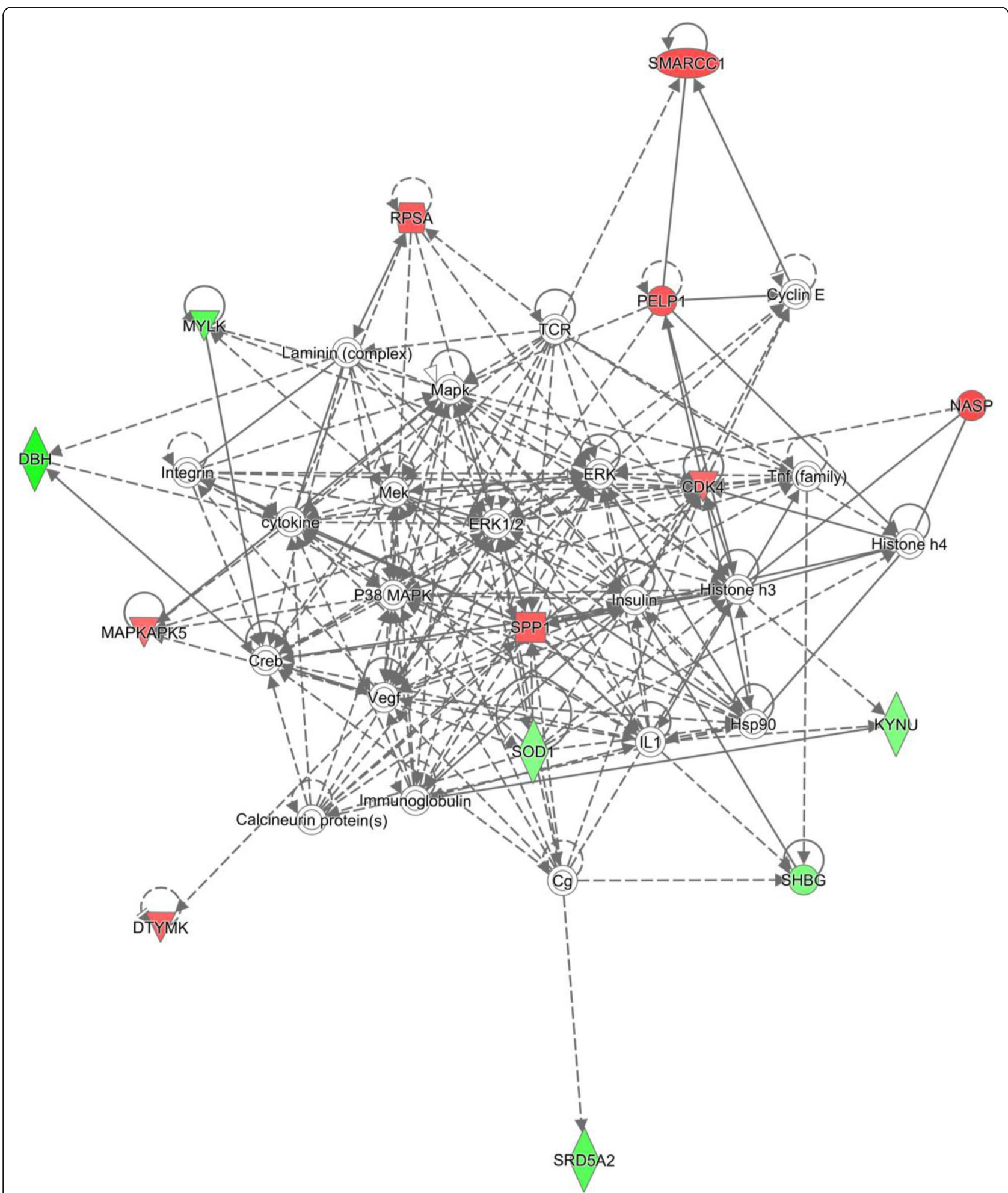

Fig. 1 The 41 mRNAs biomarkers involved in Endocrine System Development and Function, Molecular Transport, Small Molecule Biochemistry. Red stands for over-expressed and green for under-expressed 


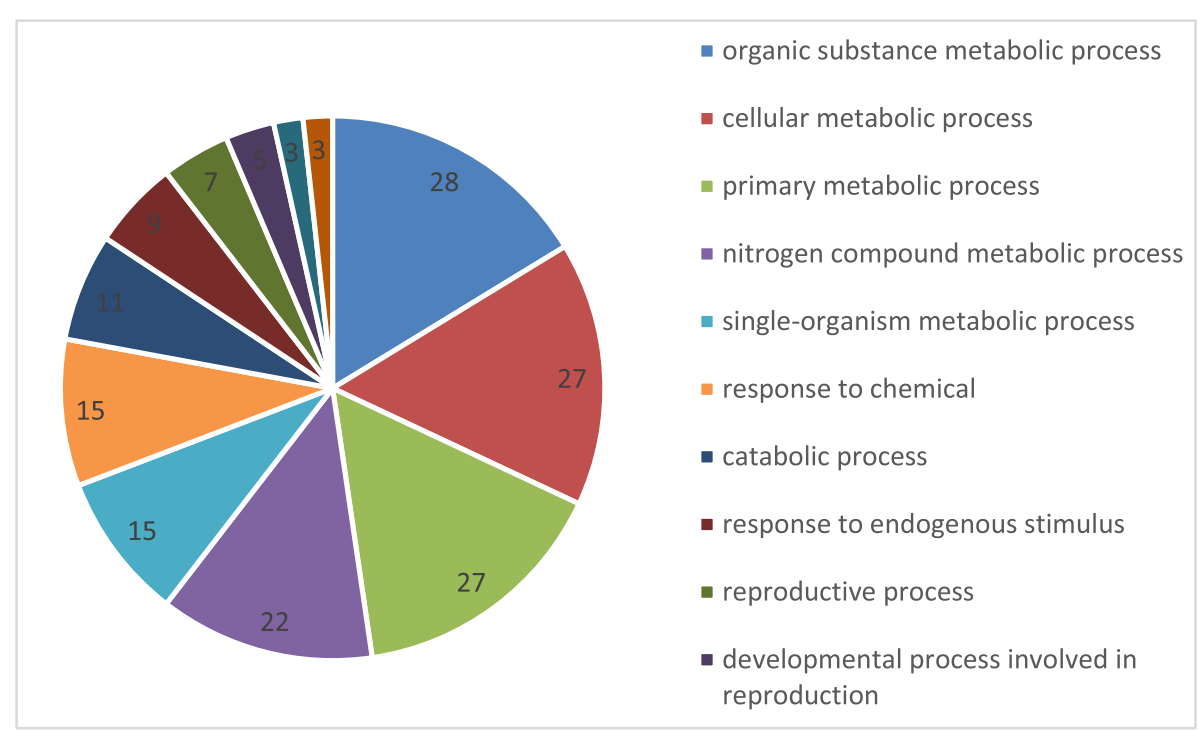

a) level 2

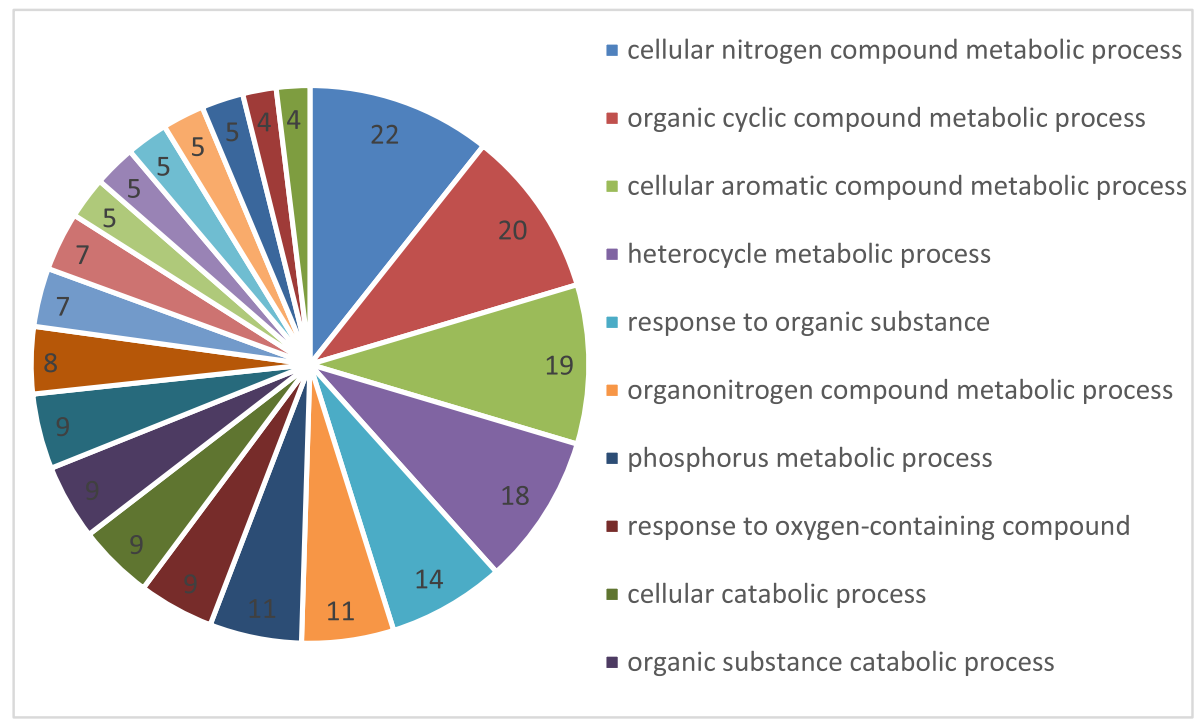

\section{b) level 3}

Fig. 2 Gene ontology biological processes enrichment analysis for 41 mRNA biomarkers. The GO terms were categorized into (a) biological processes at level 2 and (b) biological processes at level 3

$(\mathrm{AUC}=0.984, \quad$ precision $=100 \%, \quad$ accuracy $=93.8 \%$, sensitivity $=87.5 \%$, specificity $=100 \%)$ (Table 5 ).

In order to validate the prediction performance of the 41 mRNA biomarkers for cross-platform dataset, we downloaded from GEO a RNA-seq dataset (GSE94660 [26]) which contains 21 HCC samples and 21 Non-neoplastic liver samples. We used the 38 genes as variables. The gene expression data in training microarray first was averaged by the 38 genes and then was normalized to $0-1$ range by a min-max transformation function: $y=(x-\min ) /(\max -\min )$. After normalization, a SVM model with five-fold cross-validation was used for learning the training set. The Reads Per Kilobase Million (RPKM) data in testing RNA-seq containing the 38 genes was normalized using the min-max transformation and used as blind testing set. The SVM model achieved high performances $(\mathrm{AUC}=0.824$, precision $=83.3 \%$, accuracy $=$ $78.6 \%$, sensitivity $=71.4 \%$, specificity $=85.7 \%)$. The results showed that IncRNA-related and -coexpressed mRNA biomarkers had high prediction accuracy within the training and testing sets. 
Table 4 Pathway analysis for the 41 mRNA biomarkers

\begin{tabular}{|c|c|c|c|}
\hline Pathway ID & Pathway Name & Molecule & $\mathrm{AE}$ \\
\hline $1,430,728$ & Metabolism & DTYMK;DBH;HADHA;KYNU;SRD5A2 & 5 \\
\hline 109,582 & Hemostasis & F11;SOD1;SLC3A2 & 3 \\
\hline $1,640,170$ & Cell Cycle & CDK4;NUF2;TAOK1 & 3 \\
\hline 69,278 & Cell Cycle, Mitotic & CDK4;NUF2;TAOK1 & 3 \\
\hline 74,160 & Gene Expression & NR1I2;RPSA;F11 & 3 \\
\hline 162,582 & Signal Transduction & HEBP1;SPP1 & 2 \\
\hline $1,643,685$ & Disease & SPP1;RPSA & 2 \\
\hline 200,050 & Calcineurin-regulated NFAT-dependent transcription in lymphocytes & CDK4;SLC3A2 & 2 \\
\hline 200,170 & Nongenotropic Androgen signaling & SHBG;PELP1 & 2 \\
\hline 212,436 & Generic Transcription Pathway & NR112;F11 & 2 \\
\hline 382,551 & Transmembrane transport of small molecules & FLVCR1;SLC3A2 & 2 \\
\hline 453,277 & Mitotic M-M/G1 phases & NUF2;TAOK1 & 2 \\
\hline 556,833 & Metabolism of lipids and lipoproteins & HADHA;SRD5A2 & 2 \\
\hline 68,877 & Mitotic Prometaphase & NUF2;TAOK1 & 2 \\
\hline 68,886 & M Phase & NUF2;TAOK1 & 2 \\
\hline 69,306 & DNA Replication & NUF2;TAOK1 & 2 \\
\hline 71,291 & Metabolism of amino acids and derivatives & $\mathrm{DBH} ; \mathrm{KYNU}$ & 2 \\
\hline hsa00380 & Tryptophan metabolism & KYNU;HADHA & 2 \\
\hline hsa04010 & MAPK signaling pathway & MAPKAPK5;TAOK1 & 2 \\
\hline hsa04142 & Lysosome & CTSO;CTSO & 2 \\
\hline hsa04510 & Focal adhesion & SPP1;MYLK & 2 \\
\hline hsa04620 & Toll-like receptor signaling pathway & SPP1;CTSO & 2 \\
\hline
\end{tabular}

\section{Discussion}

The top four networks we identified were similar to previously reported results [27-29]. For example, De et al. used high-density oligoarrays to identify consistent differences in gene-expression between HCC and normal liver tissue. Their network analysis of differentially expressed genes classified cellular and biological functions related to regulation of gene expression and post-translational modification in HCV-related primary HCC. These included Cellular Growth and Proliferation and Cell-To-Cell Signaling and Interaction in HCV-related non HCC samples; Cellular Growth and

Table 5 Validation with SVM for the 41 mRNA biomarkers

\begin{tabular}{|c|c|c|c|c|c|c|}
\hline \multirow[t]{2}{*}{ Predicted } & \multicolumn{2}{|c|}{ Training set } & \multicolumn{2}{|c|}{ Testing set } & \multicolumn{2}{|c|}{ RNA Testing set } \\
\hline & $\mathrm{HCC}$ & Normal & $\mathrm{HCC}$ & Normal & $\mathrm{HCC}$ & Normal \\
\hline $\mathrm{HCC}$ & 7 & 0 & 7 & 0 & 15 & 3 \\
\hline normal & 1 & 8 & 1 & 8 & 6 & 18 \\
\hline Precision & $100 \%$ & & $100 \%$ & & $83.3 \%$ & \\
\hline Accuracy & $93.8 \%$ & & $93.8 \%$ & & $78.6 \%$ & \\
\hline Sensitivity & $87.5 \%$ & & $87.5 \%$ & & $71.4 \%$ & \\
\hline Specificity & $100 \%$ & & $100 \%$ & & $85.7 \%$ & \\
\hline AUC & 1 & & 0.984 & & 0.824 & \\
\hline
\end{tabular}

Proliferation and Cell Cycle in metastasis [30]. Xu et al. combined Chromatin immunoprecipitation (ChIP) on chip along with gene expression microarrays to create a genome-wide scale map of TFCP2 targets as well as the molecular function and pathways regulated by TFCP2 in HCC. They found that TFCP2-ChIP targets in SK-HEP-1 were functionally associated with cancer, cell movement, cell cycle, cell-to-cell signaling and interaction, cellular growth and proliferation [28]. Das et al. performed gene expression profiling between two groups of patients with $\mathrm{HCV}$ : one with $\mathrm{HCC}$ recurrence and second without recurrent HCC and revealed 194 differentially regulated genes between the two groups. They found that under-expressed genes were associated not only with HCC recurrence, but also with regulation of the innate immune response, cell-to-cell signaling and interaction, and the inflammatory response [29].

The Signaling, Disease, Metabolism, Cell Cycle, Immune system, and Gene Expression pathways linked with the 41 mRNA biomarkers were also reported in previous findings [22-25]. For example, two main pathogenic mechanisms were involved during hepatocarcinogenesis: (1) cirrhosis associated with hepatic regeneration after tissue damage caused by hepatitis infection, toxins or metabolic influences, and (2) mutations 
occurring in single or multiple oncogenes or tumor suppressor genes. Both mechanisms were linked in several important cellular signaling pathways. These signal pathways are of interest from a therapeutic perspective, because targeting them might help to reverse, delay or prevent tumorigenesis [24]. Numerous signaling modules including some related to growth factor signaling (e.g., IGF, EGF, PDGF, FGF, HGF), cell differentiation (WNT, Hedgehog, Notch), and angiogenesis (VEGF) have become a major source of targets for novel therapies in HCC. Different molecular mechanisms have been shown to induce aberrant pathway activation, such as point mutations, chromosomal aberrations, and epigenetically driven down-regulation [25]. Huang et al. investigated the role of EGF-EGFR signaling pathway in the development of human hepatocellular carcinoma (HCC) inflammatory environment by measuring the gene profiles of inflammatory cytokines from HCC. They found that HCC proliferation, metastasis and production of inflammatory cytokines were regulated via EGF-EGFR signaling pathways, which represent potential therapeutic targets for HCC [23].

Some biomarkers and their association with $\mathrm{HCC}$ already have been reported. For example, CDK4 (Cyclin Dependent Kinase 4) has been implicated in a number of cancer types. Jin et al. demonstrated the activation of cdk4 triggers and inhibitors of cdk4 for the prevention/ treatment of Non-alcoholic Fatty Liver Disease [31]. Secreted phosphoprotein-1 (SPP1) was found to be overexpressed in metastatic hepatocellular carcinoma (HCC), and had potential to act as both a diagnostic marker and a therapeutic target for HCC [32]. Li et al. found that downregulation of Superoxide Dismutase 1 (SOD1) was correlated with histopathological grading and might be a good candidate gene for HCC [33].

LncRNA/mRNA expression profiling has been widely used for biomarker discovery of cancers, for example, liver cancer, gastric cancer, bladder cancer, colon cancer, pancreatic cancer, laryngeal cancer, and colorectal cancer. This is because LncRNAs is important in identifying biomarkers for various human cancers. Unraveling the co-expression pattern between mRNAs and lncRNAs can further help researchers better understand the mechanism of various human cancers. The IncRNA-related and -coexpressed method to detect mRNA biomarkers we presented in the study can work not only for HCC but also for all other human cancers.

The advantage of the lncRNA-related and -coexpressed method to detect mRNA biomarkers is that we consider the co-expression between mRNA and lncRNA, filter out some unimportant mRNAs and lncRNAs by setting significant threshold, and focus on the most important mRNAs and lncRNAs and their coexpressed networks. Compared with the original findings from the datasets
(GSE58043, GSE89186, GSE64631, and GSE55191, http:// www.ncbi.nlm.nih.gov/geo) [14-16], it shows that we found the Endocrine System Development and Function as top network associated with liver cancer. This finding is consistent with the fact that liver abnormalities have strong association with endocrine diseases [34, 35]. It would be difficult to discover the Endocrine System Development and Function as top network without using lncRNAs' coexpression as one kind of filter. As shown in the result section, there are 3543 significantly differentially expressed mRNA biomarkers at first. It is the lncRNA coexpression filter in our method that shortens the number of biomarker candidates and unravels the final 41 mRNAs (38 genes) which functionally link to the Endocrine System Development and Function.

There is a limitation of the study with small sample size. We have tried our best to collect all the four available LncRNA/mRNA expression profiling related to HCC [14-16]. In the future, we will continue to collect HCC LncRNA/mRNA expression profiling data with our collaborators.

\section{Conclusion}

We developed a lncRNA-related and -coexpressed method to detect mRNA biomarkers with HCC. Top networks such as "Endocrine System Development and Function, Molecular Transport, Small Molecule Biochemistry" and enriched pathways such as Cell Cycle, Signaling, Metabolism, and Immune System were also discovered. Unraveling these intricate networks and pathways is essential to understanding the biological mechanisms of HCC development and progression. Our method has the potential to provide a basis for biomarker identification in $\mathrm{HCC}$ or other diseases.

\section{Abbreviation}

AUC: Area Under the Curve; ChIP: Chromatin immunoprecipitation; DAVID: the Database for Annotation, Visualization and Integrated Discovery; HCC: Hepatocellular Carcinoma; IPA: Ingenuity Pathway Analysis; IPAD: Integrated Pathway Analysis Database; IncRNA: Long noncoding RNA; RPKM: The Reads Per Kilobase Million; SVM: Support Vector Machine

\section{Acknowledgements}

Not applicable.

\section{Funding}

This work was supported by an Institutional Development Award (IDeA) from the National Institute of General Medical Sciences of the National Institutes of Health under grant number P20GM103449. Publication of this article was sponsored by P20GM103449 grant.

\section{Availability of data and materials}

Cao SW, Huang JL, Chen J, Hu YW, Hu XM, Ren TY, Zheng SH, Lin JD, Tang J, Zheng $L$ et al.: Long non-coding RNA UBE2CP3 promotes tumor metastasis by inducing epithelial-mesenchymal transition in hepatocellular carcinoma. Oncotarget 2017. https://dx.doi.org/10.18632\%2Foncotarget.18524. Peng H, Ishida M, Li L, Saito A, Kamiya A, Hamilton JP, Fu R, Olaru AV, An F, Popescu I et al.: Pseudogene INTS6P1 regulates its cognate gene INTS6 through competitive binding of miR-17-5p in hepatocellular carcinoma. Oncotarget 2015. https://dx.doi.org/10.18632\%2Foncotarget.3290. 
Chen J, Fu Z, Ji C, Gu P, Xu P, Yu N, Kan Y, Wu X, Shen R, Shen Y: Systematic gene microarray analysis of the IncRNA expression profiles in human uterine cervix carcinoma. Biomed Pharmacother 2015. https://doi.org/10.1016/ j.biopha.2015.04.010.

Yoo S, Wang W, Wang Q, Fiel MI, Lee E, Hiotis SP, Zhu J: A pilot systematic genomic comparison of recurrence risks of hepatitis B virus-associated hepatocellular carcinoma with low- and high-degree liver fibrosis. BMC Med 2017. https://doi.org/10.1186/s12916-017-0973-7.

\section{About this supplement}

This article has been published as part of BMC Medical Genomics Volume 12 Supplement 1, 2019: Selected articles from the International Conference on Intelligent Biology and Medicine (ICIBM) 2018: medical genomics. The full contents of the supplement are available online at https:// bmcmedgenomics.biomedcentral.com/articles/supplements/volume-12supplement-1.

\section{Authors' contributions}

FZ conceived the initial work and designed the method. FZ developed the prediction method, and performed the computational analyses. RB proofread the manuscript. LC collected testing dataset for validating the biomarkers. LD and BD performed a literature review. All authors are involved in the drafting and revisions of the manuscript. All of the authors have read and approved the final manuscript.

\section{Ethics approval and consent to participate}

Not applicable.

\section{Consent for publication}

Not applicable.

\section{Competing interests}

The authors declare that they have no competing interests.

\section{Publisher's Note}

Springer Nature remains neutral with regard to jurisdictional claims in published maps and institutional affiliations.

\section{Author details}

'Vermont Genetics Network, University of Vermont, Burlington, VT 05405, USA. ${ }^{2}$ Department of Biology, University of Vermont, Burlington, VT 05405. USA. ${ }^{3}$ School of Medicine, University of California, San Diego, 9500 Gilman Drive, La Jolla, CA 92093-0606, USA. ${ }^{4}$ Department of Neurosciences, School of Medicine, University of California, San Diego, 9500 Gilman Drive \#0949, La Jolla, CA 92093, USA. 5 Department of Pharmacology and Neuroscience, University of North Texas Health Science Center, Fort Worth, TX, USA.

\section{Published: 31 January 2019}

\section{References}

1. Kim HN, Kim BH, Cho J, Ryu S, Shin H, Sung J, Shin C, Cho NH, Sung YA Choi BO, et al. Pathway analysis of genome-wide association datasets of personality traits. Genes Brain Behav. 2015;14(4):345-56.

2. What are the key statistics about liver cancer? [http://www.cancer.org/ cancer/livercancer/detailedguide/liver-cancer-what-is-key-statistics], date accessed: 10/16/2018

3. Khalaf N, Ying J, Mittal S, Temple S, Kanwal F, Davila J, El-Serag HB. Natural history of untreated hepatocellular carcinoma in a US cohort and the role of Cancer surveillance. Clin Gastroenterol Hepatol. 2017;15(2):273-81 e271.

4. Lin N, Dang J, Rana TM. Haunting the HOXA locus: two faces of IncRNA regulation. Cell Stem Cell. 2015;16(5):449-50.

5. Yin $Y$, Yan $P$, Lu J, Song G, Zhu Y, Li Z, Zhao Y, Shen B, Huang X, Zhu H, et al. Opposing roles for the IncRNA haunt and its genomic locus in regulating HOXA gene activation during embryonic stem cell differentiation. Cell Stem Cell. 2015:16(5):504-16.

6. Lu KH, Li W, Liu XH, Sun M, Zhang ML, Wu WQ, Xie WP, Hou YY. Long noncoding RNA MEG3 inhibits NSCLC cells proliferation and induces apoptosis by affecting p53 expression. BMC Cancer. 2013;13.

7. Mondal T, Subhash S, Vaid R, Enroth S, Uday S, Reinius B, Mitra S, Mohammed A, James AR, Hoberg E, et al. MEG3 long noncoding RNA regulates the TGF-beta pathway genes through formation of RNA-DNA triplex structures. Nat Commun. 2015;6.

8. Zhou YL, Zhang X, Klibanski A. MEG3 noncoding RNA: a tumor suppressor. J Mol Endocrinol. 2012:48(3):R45-53.

9. Zhao Y, Guo Q, Chen J, Hu J, Wang S, Sun Y. Role of long non-coding RNA HULC in cell proliferation, apoptosis and tumor metastasis of gastric cancer: a clinical and in vitro investigation. Oncol Rep. 2014;31(1):358-64.

10. Sun $X H$, Yang LB, Geng XL, Wang $R$, Zhang ZC. Increased expression of InCRNA HULC indicates a poor prognosis and promotes cell metastasis in osteosarcoma. Int J Clin Exp Pathol. 2015;8(3):2994-3000.

11. Clark MB, Johnston RL, Inostroza-Ponta M, Fox AH, Fortini E, Moscato $P$, Dinger ME, Mattick JS. Genome-wide analysis of long noncoding RNA stability. Genome Res. 2012:22(5):885-98.

12. Askarian-Amiri ME, Crawford J, French JD, Smart CE, Smith MA, Clark MB, Ru K, Mercer TR, Thompson ER, Lakhani SR, et al. SNORD-host RNA Zfas1 is a regulator of mammary development and a potential marker for breast cancer. Rna. 2011;17(5):878-91.

13. Li T, Xie J, Shen C, Cheng D, Shi Y, Wu Z, Deng X, Chen H, Shen B, Peng C, et al. Amplification of long noncoding RNA ZFAS1 promotes metastasis in hepatocellular carcinoma. Cancer Res. 2015;75(15):3181-91.

14. Cao SW, Huang JL, Chen J, Hu YW, Hu XM, Ren TY, Zheng SH, Lin JD, Tang J, Zheng L, et al. Long non-coding RNA UBE2CP3 promotes tumor metastasis by inducing epithelial-mesenchymal transition in hepatocellular carcinoma. Oncotarget. 2017;8(39):65370-85.

15. Peng H, Ishida M, Li L, Saito A, Kamiya A, Hamilton JP, Fu R, Olaru AV, An F, Popescu I, et al. Pseudogene INTS6P1 regulates its cognate gene INTS6 through competitive binding of miR-17-5p in hepatocellular carcinoma. Oncotarget. 2015;6(8):5666-77.

16. Chen J, Fu Z, Ji C, Gu P, Xu P, Yu N, Kan Y, Wu X, Shen R, Shen Y. Systematic gene microarray analysis of the IncRNA expression profiles in human uterine cervix carcinoma. Biomed Pharmacother. 2015;72:83-90.

17. Kim S, Chen MH, Ibrahim JG, Shah AK, Lin J. Bayesian inference for multivariate meta-analysis box-cox transformation models for individual patient data with applications to evaluation of cholesterol-lowering drugs. Stat Med. 2013;32(23):3972-90.

18. Zhang F, Chen JY. Discovery of pathway biomarkers from coupled proteomics and systems biology methods. BMC Genomics. 2010;11(Suppl 2):S12.

19. Witt PL, McGrain P. Comparing two sample means t tests. Phys Ther. 1985; 65(11):1730-3.

20. Huang DW, Sherman BT, Lempicki RA. Systematic and integrative analysis of large gene lists using DAVID bioinformatics resources. Nat Protoc. 2009;4(1):44-57.

21. Zhang F, Drabier R: IPAD: the Integrated Pathway Analysis Database for Systematic Enrichment Analysis. BMC Bioinformatics. 2012;13:Suppl 15:S7

22. Mauriz JL, Tunon MJ, Gonzalez-Gallego J. Apoptotic signaling pathways as a target for the treatment of liver diseases. Mini reviews in medicinal chemistry. 2008;8(14):1485-93.

23. Huang $P, X u X$, Wang $L$, Zhu B, Wang $X$, Xia J. The role of EGF-EGFR signalling pathway in hepatocellular carcinoma inflammatory microenvironment. J Cell Mol Med. 2014;18(2):218-30.

24. Whittaker S, Marais R, Zhu AX. The role of signaling pathways in the development and treatment of hepatocellular carcinoma. Oncogene. 2010:29(36):4989-5005

25. Moeini A, Cornella H, Villanueva A. Emerging signaling pathways in hepatocellular carcinoma. Liver cancer. 2012;1(2):83-93.

26. Yoo S, Wang W, Wang Q, Fiel MI, Lee E, Hiotis SP, Zhu J. A pilot systematic genomic comparison of recurrence risks of hepatitis $B$ virus-associated hepatocellular carcinoma with low- and high-degree liver fibrosis. BMC Med. 2017;15(1):214

27. Bandres-Ciga S, Mencacci NE, Duran R, Barrero FJ, Escamilla-Sevilla F, Morgan S, Hehir J, Vives F, Hardy J, Pittman AM: Analysis of the genetic variability in Parkinson's disease from Southern Spain. Neurobiol Aging. 2016;37:210e211-210 e215.

28. Xu X, Liu Z, Zhou L, Xie H, Cheng J, Ling Q, Wang J, Guo H, Wei X, Zheng S. Characterization of genome-wide TFCP2 targets in hepatocellular carcinoma: implication of targets FN1 and TJP1 in metastasis. J Exp Clin Cancer Res : CR. 2015;34:6.

29. Das T, Diamond DL, Yeh M, Hassan S, Bryan JT, Reyes JD, Perkins JD. Molecular signatures of recurrent hepatocellular carcinoma secondary to hepatitis C virus following liver transplantation. J Transplantat. 2013;2013:878297. 
30. De Giorgi V, Buonaguro L, Worschech A, Tornesello ML, Izzo F, Marincola FM, Wang E, Buonaguro FM. Molecular signatures associated with HCVinduced hepatocellular carcinoma and liver metastasis. PLoS One. 2013;8(2):e56153.

31. Jin J, Valanejad L, Nguyen TP, Lewis K, Wright M, Cast A, Stock L, Timchenko $\mathrm{L}$, Timchenko NA. Activation of CDK4 triggers development of non-alcoholic fatty liver disease. Cell Rep. 2016;16(3):744-56.

32. Shin HD, Park BL, Cheong HS, Yoon JH, Kim YJ, Lee HS. SPP1 polymorphisms associated with HBV clearance and HCC occurrence. Int J Epidemiol. 2007;36(5):1001-8.

33. Li Y, Wan D, Wei W, Su J, Cao J, Qiu X, Ou C, Ban K, Yang C, Yue H. Candidate genes responsible for human hepatocellular carcinoma identified from differentially expressed genes in hepatocarcinogenesis of the tree shrew (Tupaia belangeri chinesis). Hepatol Res. 2008;38(1):85-95.

34. Handzlik-Orlik G, Krysiak R, Kedzierski L, Okopien B. Endocrine abnormalities in liver diseases. Przegl Lek. 2016;73(5):324-33.

35. Burra P. Liver abnormalities and endocrine diseases. Best Pract Res Clin Gastroenterol. 2013;27(4):553-63.

Ready to submit your research? Choose BMC and benefit from:

- fast, convenient online submission

- thorough peer review by experienced researchers in your field

- rapid publication on acceptance

- support for research data, including large and complex data types

- gold Open Access which fosters wider collaboration and increased citations

- maximum visibility for your research: over $100 \mathrm{M}$ website views per year

At $\mathrm{BMC}$, research is always in progress.

Learn more biomedcentral.com/submissions 